\title{
Role of vascular endothelial growth factor receptor-3/Flt-4 in early-stage cervical cancer
}

\author{
SHIQIAN ZHANG ${ }^{1}$, HAO YU ${ }^{2}$ and LINLIN ZHANG ${ }^{3}$ \\ ${ }^{1}$ Department of Obstetrics and Gynecology, Qilu Hospital of Shandong University, Ji'nan 250012; \\ ${ }^{2}$ Department of Gynecological Oncology, Cancer Research Institute of Shandong Province, Ji'nan 250117; \\ ${ }^{3}$ Department of Obstetrics and Gynecology, Fourth People's Hospital of Jinan, Ji'nan 250031, P.R. China
}

Received November 3, 2009; Accepted March 19, 2010

DOI: $10.3892 / o 1 \_00000080$

\begin{abstract}
The present study was designed to investigate the role of vascular endothelial growth factor receptor (VEGFR)-3/ Flt-4 in the early stages of cervical cancer. VEGFR-3/Flt-4 expression, vascular endothelial growth factor (VEGF)-C and VEGF-D in the early stages (Ia-IIa) of cervical cancer in 41 patients was examined by immunohistochemical analysis. Additionally, the VEGFR-3/Flt-4-marked vascular density (MVD) was examined and the relationship of these factors with clinicopathological factors was analyzed. VEGFR-3/ Flt-4 was found to be expressed in lymphatic endothelial cells and, to a certain extent, in vascular endothelial cells. The VEGFR-3/Flt-4-positive vessels were largely distributed in the stroma surrounding the tumor tissues, and these vessels were morphologically divided into blood and lymphatic vessels, respectively. Cancer cells were found to express VEGF-C, VEGF-D and VEGFR-3/Flt-4, and their positive expression rate was $48.7 \%$ (20/41), 58.5\% (24/41) and $63.4 \%$ (26/41), respectively. VEGFR-3/Flt-4 expression in the cancer cells of the cervical cancer patients in our study was found to be correlated to the clinical stage, lymph node metastasis, lymphatic invasion and expression of VEGF-C and VEGF-D. However, this expression was unrelated to menstrual status, histological grade and histological classification. MVD was correlated to the clinical stage and expression of VEGF-C and VEGF-D, but was unrelated to menstrual status, histological grade, histological classification, lymph node metastasis and lymphatic invasion. In conclusion, VEGFR-3/Flt-4 plays an important role in the early stages of cervical cancer.
\end{abstract}

Correspondence to: Dr Shiqian Zhang, Department of Obstetrics and Gynecology, Qilu Hospital of Shandong University, Wenhuaxi Road 107, Ji'nan 250012, Shandong, P.R. China

E-mail: zhangshiqian370112@126.com

Key words: cervical cancer, vascular endothelial growth factor receptor-3/Flt-4, vascular endothelial growth factor-C, vascular endothelial growth factor-D

\section{Introduction}

Vascular endothelial growth factor receptor (VEGFR)-3 (also known as Flt-4) is a tyrosine kinase (TK) receptor of vascular endothelial growth factor (VEGF)-C and VEGF-D. It is mainly expressed in lymphatic endothelial cells, but also in neovascular endothelial cells of the liver, splenic sinusoid, trauma repair tissues and tumor tissues. Studies showed that cancer cells express VEGFR-3/Flt-4, which contributes to malignant tumor progression in various ways (1). In this study, an immunohistochemical streptavidin-peroxidase (SP) method was used to detect the expression of VEGF-C, VEGF-D and VEGFR-3/ Flt-4 in early-stage cervical cancer tissues. The VEGFR-3/ Flt-4-marked vascular density (MVD) was also determined. The relationship of these factors with clinicopathological factors was analyzed, as well as the influence of VEGFR-3/ Flt-4 in the early stages of cervical cancer.

\section{Materials and methods}

Subjects. This study was carried out following international and national regulations, and informed consent was obtained from all of the patients. Cervical cancer tissues were extracted from the 41 patients included in the study by gynecologic surgery between September 2007 and December 2008 at the Qilu Hospital of Shandong University. The tumor tissues were fixed in $4 \%$ paraformaldehyde and embedded in paraffin. The patients did not undergo any pre-operative chemotherapy, but a definite diagnosis of cervical cancer was established by postoperative pathology. Clinical staging was performed according to the International Federation of Gynecology and Obstetrics (FIGO, 2000) staging system: stage Ia was found in 7 cases, stage Ib in 14 cases and stage IIa in 20 cases. The histological grading revealed G1 in 7 cases, G2 in 13 cases and G3 in 21 cases. The histological classification showed 37 tumors to be squamous cell carcinomas and 4, adenocarcinomas. Lymph node metastasis was found in 15 patients and non-lymph node metastasis in 26 patients. The patients were 26-70 years of age (median 42). Additionally, 12 patients had developed cancer at menopause and 29 prior to menopause. Microscopic examination of the cancer cells from the lymphatic lumen confirmed lymphatic invasion in 6 cases and non-lymphatic invasion in 35 cases. 

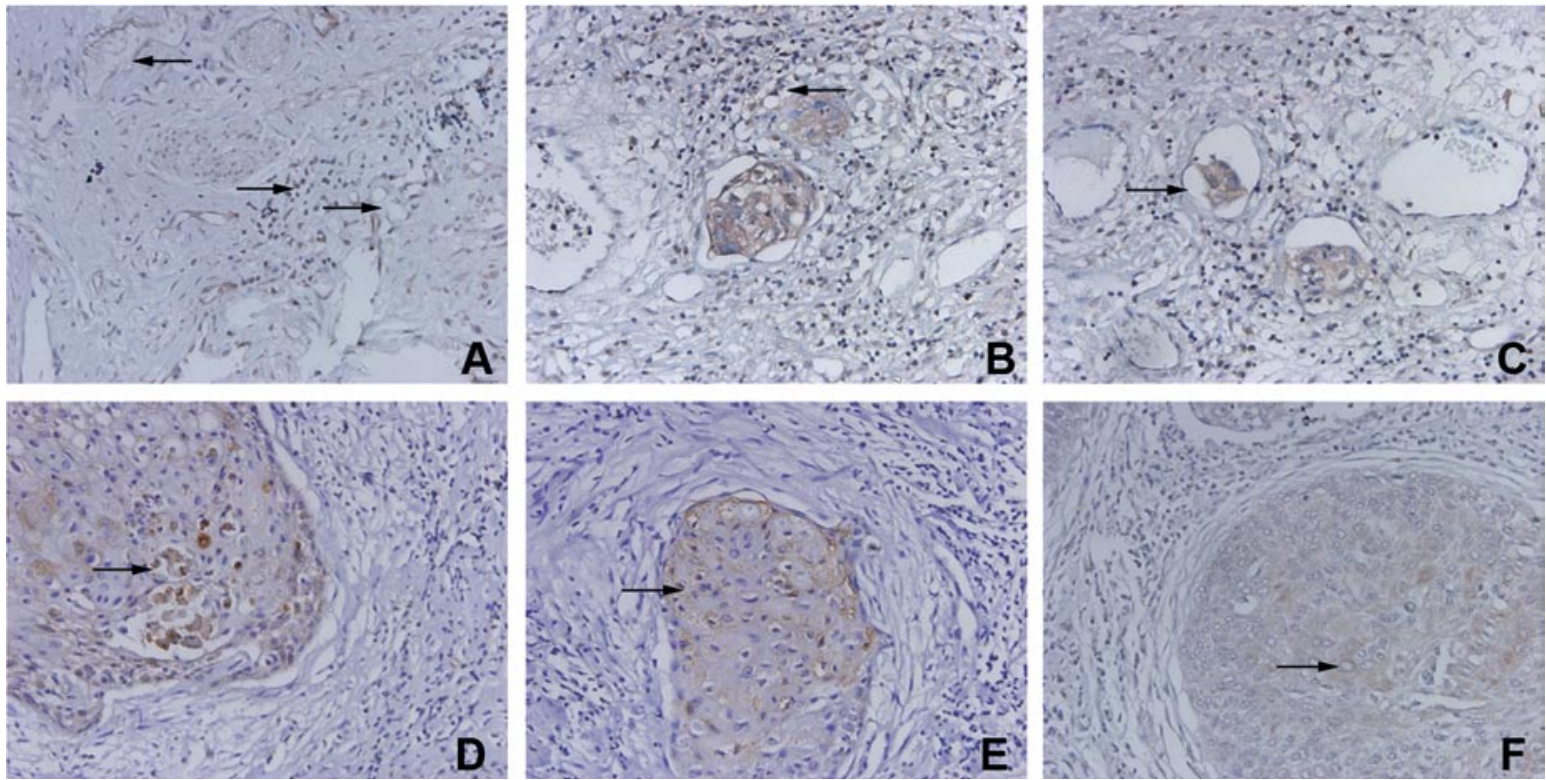

Figure 1. VEGFR-3/Flt-4 expression in early-stage cervical cancer tissues. (A) VEGFR-3/Flt-4 is expressed in inflammatory cells. (B) VEGFR-3/Flt-4positive vessels were distributed in the stroma. (C) Cancer cells were found in VEGFR-positive vessels. (D-F) Cancer cells were found to express VEGFR-C, VEGFR-D and VEGFR-3/Flt-4.

Immunohistochemical SP staining. The immunohistochemical SP staining kit (LHK612; Jingmei Bioengineering Co., Ltd.) was used in this study strictly according to the instructions provided by the manufacturer. The paraffin-embedded tissues were sliced into 5- $\mu \mathrm{m}$ sections and treated. After conventional dewaxing with xylene and rehydration, microwave antigen retrieval was performed at $95^{\circ} \mathrm{C}$ for $10 \mathrm{~min}$ using a citrate buffer ( $\mathrm{pH} \mathrm{6.0)}$ as the antigen retrieval buffer. This solution was incubated with a hydrogen peroxide (3\%) and methanol solution for $10 \mathrm{~min}$ to block the endogenous peroxidase. A blocking solution containing $10 \%$ goat serum was used to block non-specific antibodies. Certain polyclonal antibodies were used as the first antibody, including rabbit anti-human VEGF-C (anti-VEGF-C, ZA-0266; Beijing Zhongshan Jinqiao Biotechnology Co., Ltd.) at a dilution of 1:50, rabbit antihuman VEGF-D (anti-VEGF-D, BA1461; Wuhan Boster Co., Ltd.) at a dilution of 1:100 and rabbit anti-human VEGFR-3/ Flt-4 (anti-VEGFR-3/Flt-4, ab27278; Abcam Ltd., UK) at a dilution of 1:200. After incubation for $60 \mathrm{~min}$, anti-rabbit biotinylated secondary antibody was added, and the mixture was incubated for $30 \mathrm{~min}$. Horseradish peroxidase-labeled streptavidin was added, and the mixture was incubated for another $30 \mathrm{~min}$. Staining with 3,3'-diaminobenzidine substrate and restaining with hematoxylin were performed. After the slides containing the sections with neutral gum were sealed, the stained sections were microscopically examined. In this experiment, the control was treated in the same manner, except that PBS buffer was used instead of the first antibody as a negative control, and the sections of breast cancer tissue were used as a positive control.

Determinations. The appearance of yellowish-brown granules in the cytoplasm indicated positive results for VEGF-C, VEGF-D and VEGFR-3/Flt-4 staining. On the basis of the method reported by Jüttner et al (2) and the proportion of positive cells, the results were classified as: -, no positive cells; ,$+ 0-5 \%$ positive cells;,$++ 5-50 \%$ positive cells;,$+++>50 \%$ positive cells), with ++ and +++ being considered a positive expression. MVD was determined according to the method reported by Weidner et al (3). Briefly, the dense-staining zones ('hot spots') of the marker-positive vessel lumens were microscopically observed. The number of positive lumens in the scope of one viewing field were counted at a high magnification, and the mean value of the five numbers (from five viewing fields) of the marker-positive lumens at high magnification represented MVD.

Measurement data were analyzed using the statistical software SPSS 13.0. MVD is expressed as the mean \pm SD. $P<0.05$ indicates that the differences were statistically significant.

\section{Results}

Expression of VEGFR-3/Flt-4 in early-stage cervical cancer tissues. VEGFR-3/Flt-4 was found to be expressed in lymphatic endothelial cells and in certain vascular endothelial cells. VEGFR-3/Flt-4-positive vessels were morphologically divided into blood and lymphatic vessels. VEGFR-3/Flt-4 was also expressed in certain inflammatory cells around the VEGFR-3/Flt-4-positive vessels (Fig. 1A). Some VEGFR-3/ Flt-4-positive vessels were mainly distributed in the stroma surrounding the tumor tissues (Fig. 1B). Cancer cells were found in some VEGFR-positive vessels (Fig. 1C). Cancer cells were found to express VEGF-C, VEGF-D and VEGFR-3/ Flt-4, and their positive expression rate was $48.7 \%$ (20/41), $58.5 \%(24 / 41)$ and $63.4 \%$ (26/41), respectively (Fig. 1D-F).

Respective correlation of VEGFR-3/Flt-4 and MVD with clinicopathological factors, VEGF-C and VEGF-D in earlystage cervical cancer tissues. VEGFR-3/Flt-4 expression in the cancer cells of patients with cervical cancer was found 
Table I. Respective correlation of VEGFR-3/Flt-4 and marked vascular density (MVD) with clinicopathological factors, VEGF-C and VEGF-D in early-stage cervical cancer tissues.

\begin{tabular}{|c|c|c|c|c|c|c|}
\hline \multirow[t]{2}{*}{ Clinicopathological factors } & \multirow[t]{2}{*}{$\mathrm{n}$} & \multicolumn{3}{|c|}{ VEGFR-3/Flt-4 } & \multirow[t]{2}{*}{ MVD [mean (SD)] } & \multirow[t]{2}{*}{ P-value } \\
\hline & & $(+)$ & $(-)$ & P-value & & \\
\hline \multicolumn{7}{|l|}{ Menstrual status } \\
\hline Pre-menopausal & 29 & 19 & 10 & NS & $25.97(1.48)$ & NS \\
\hline Menopausal & 12 & 7 & 5 & & $25.41(1.83)$ & \\
\hline \multicolumn{7}{|l|}{ Clinical stages } \\
\hline Ia & 7 & 3 & 4 & $<0.01$ & $23.29(0.49)$ & $<0.01$ \\
\hline $\mathrm{Ib}$ & 14 & 5 & 9 & & $25.21(0.43)$ & \\
\hline IIa & 20 & 18 & 2 & & $27.10(0.85)$ & \\
\hline \multicolumn{7}{|l|}{ Histological grade } \\
\hline G1 & 7 & 4 & 3 & NS & $25.43(1.98)$ & NS \\
\hline G2 & 13 & 8 & 5 & & $26.08(1.75)$ & \\
\hline G3 & 21 & 12 & 9 & & $25.76(1.37)$ & \\
\hline \multicolumn{7}{|l|}{ Lymph node metastasis } \\
\hline No & 26 & 12 & 14 & $<0.05$ & $25.50(1.84)$ & NS \\
\hline Yes & 15 & 14 & 1 & & $26.33(0.82)$ & \\
\hline \multicolumn{7}{|l|}{ Lymphatic invasion } \\
\hline No & 35 & 20 & 15 & $<0.05$ & $25.69(1.66)$ & NS \\
\hline Yes & 6 & 6 & 0 & & $26.50(0.84)$ & \\
\hline \multicolumn{7}{|l|}{ Histological classification } \\
\hline Squamous cell carcinoma & 37 & 23 & 14 & NS & 25.78 (1.64) & NS \\
\hline Adenocarcinoma & 4 & 3 & 1 & & $26.00(1.15)$ & \\
\hline \multicolumn{7}{|l|}{ VEGF-C } \\
\hline$(+)$ & 20 & 17 & 3 & $<0.01$ & $24.50(1.00)$ & $<0.01$ \\
\hline$(-)$ & 21 & 9 & 12 & & $27.05(0.86)$ & \\
\hline \multicolumn{7}{|l|}{ VEGF-D } \\
\hline$(+)$ & 24 & 20 & 4 & $<0.01$ & $25.08(1.38)$ & $<0.01$ \\
\hline$(-)$ & 17 & 6 & 11 & & $26.82(1.28)$ & \\
\hline
\end{tabular}

NS, no statistical significance.

to be correlated to the clinical stage, lymph node metastasis, lymphatic invasion and the expression of VEGF-C and VEGF-D. The expression, however, was unrelated to menstrual status, histological grade and histological classification. MVD was correlated to the clinical stage and the expression of VEGF-C and VEGF-D, but was unrelated to menstrual status, histological grade, histological classification, lymph node metastasis and lymphatic invasion (Table I).

\section{Discussion}

Members of the vascular endothelial growth factor families (VEGF-A-E) and their receptors (VEGFR-1-3) have been the focus of research owing to their ability to promote angiogenesis or lymphangiogenesis. In particular, this study involved the analysis of the mechanisms of the growth factor family in the promotion of malignant tumor growth and metastasis, and the corresponding gene therapy strategies. Current research has shown that tumor angiogenesis is mainly regulated by the
VEGF-A/VEGFR-2 system and that tumor lymphangiogenesis is mainly regulated by the VEGF-C, -D/VEGFR-3 (Flt-4) system (4). The results of our study showed that VEGFR-3/ Flt-4 was expressed in lymphatic endothelial cells and, to a certain extent, in vascular endothelial cells with VEGFR-3/ Flt-4-positive vessels being divided into blood and lymphatic vessels. VEGFR-3/Flt-4-positive vascular density (MVD) was found to be correlated to the clinical stage of the cancers and the expression of VEGF-C and VEGF-D, but was unrelated to menstrual status, histological grade and other clinicopathological factors. These results differed from Yasuoka et al (5) due to the fact that VEGFR-3/Flt-4 plays an important role in the regulation of tumor lymphangiogenesis and angiogenesis. VEGF-C and VEGF-D are secreted by cancer cells and show a paracrine action on the TK receptor (VEGFR-3/ Flt-4), which is expressed in lymphatic and vascular endothelial cells to mediate proliferation and differentiation of endothelial cells and lumen formation. The increase in the number of blood vessels that supply tumor tissues provides 
essential nutrients for cancer cell growth, while an increase in the number of lymphatic vessels in tumor tissues provides channels for lymphatic invasion and metastasis of cancer cells and facilitates their transfer and spread. However, there is a lack of accurate lymphatic endothelial markers which differ according to the type, position and stages of cancers for lymphatic vessels (6). Therefore, the exact mechanism underlying VEGFR-3/Flt-4 action on tumor angiogenesis and lymphangiogenesis warrants further investigation.

VEGFR-3/Flt-4 is mainly expressed in endothelial cells as a TK receptor of VEGF-C and VEGF-D. In addition, studies have shown that numerous cancer cells express Flt-4 (i.e., VEGFR-3), which plays an important role in tumor progression. Clinical trials in many human malignant tumors have shown that VEGFR-3 expression in cancer cells is correlated to the clinical stage of cancer in patients, the degree of cell differentiation, lymph node metastasis and patient prognosis (7-9). Van Trappen et al reported that VEGFR-3/Flt-4 expression levels changed with the progression of cervical intraepithelial carcinoma into cervical cancer (10). Therefore, it is speculated that VEGFRs may be involved in the phenotypic transformation of carcinoma cells to promote lymphangiogenesis in cervical cancer. The results obtained by in vitro migration and cervical mucus invasion tests $(11,12)$ indicated that strong invasive cancer cell lines, such as $\mathrm{SiHa}$ and breast cancer cell lines MDA-MB-231 and Hs578T, express Flt-4 (i.e., VEGFR-3) and VEGF-C. Recombinant human VEGF-C (Cys156Ser) facilitates the migration and invasion of cancer cells. However, cellular migration and invasion were greatly reduced when recombinant Flt-4/Fc was used to block the VEGF-C receptor (VEGFR-3/Flt-4). Masood et al reported that VEGFR-3/Flt-4 promotes the growth of malignant mesothelioma (13). Dias et al reported that VEGFR-3/Flt-4 may be involved in leukemic cell proliferation, survival and chemical drug resistance (14). The results of this study showed that the VEGFR-3/Flt-4 expression of cancer cells in cervical cancer tissues was correlated to lymph node metastasis and lymphatic invasion, and the clinical stage was correlated to the expression of the VEGFR-3/Flt-4 ligands (VEGF-C and VEGF-D). The possible mechanism involved is expressed by cancer cells that secrete VEGF-C and VEGF-D which, in turn, act in an autocrine manner on VEGFR-3/Flt-4 in cancer cells to facilitate migration and invasion, thereby promoting tumor lymphatic invasion and lymph node metastasis. However, the receptors of VEGF-C and VEGF-D have been reported to be heterogeneous in different types of cancer cells, e.g., VEGF-C or VEGF-D receptors also interacted with VEGFR-2, NRP-1 and NRP-2 besides VEGFR-3/Flt-4 (12), which has led to inconsistencies in the results. For example, Jüttner et al (2) reported that the VEGFR-3/Flt-4 expression of gastric cancer cells was unrelated to lymph node metastasis in patients with gastric cancer.

The results of the present study showed that VEGFR-3/ Flt-4 was expressed in certain inflammatory cells in the stroma surrounding tumor tissues, but its role remains unknown. A previous study showed (15) that the inflammatory cells of tumor stroma may also play an important role in tumor lymphangiogenesis. In conclusion, VEGFR-3/Flt-4 may play several roles in malignant tumor progression, and its effect may vary according to the type of cancer. However, the exact mechanism underlying these roles requires further investigation.

\section{Acknowledgements}

This study was supported by the Natural Science Foundation of Shandong Province (no. Y2008C70).

\section{References}

1. Su JL, Yen CJ, Chen PS, et al: The role of the VEGF-C/VEGFR-3 ax is in cancer progression. Br J Cancer 96: 541-545, 2007.

2. Jüttner S, Wissmann C, Jons T, et al: Vascular endothelial growth factor-D and its receptor VEGFR-3: two novel independent prognostic markers in gastric adenocarcinoma. J Clin Oncol 24: 228-240, 2006.

3. Weidner N, Semple JP, Welch WR and Folkman J: Tumor angiogenesis and metastasis - correlation in invasive breast carcinoma. N Engl J Med 324: 1-8, 1991.

4. Rouzaut A, Irigoyen M and Montuenga LM: Lymphangiogenesis and lung cancer. J Thorac Oncol 2: 384-386, 2007.

5. Yasuoka H, Nakamura Y, Zuo H, et al: VEGF-D expression and lymph vessels play an important role for lymph node metastasis in papillary thyroid carcinoma. Mod Pathol 18: 1127-1133, 2005.

6. Kawai Y, Minami T, Fujimori M, et al: Characterization and microarray analysis of genes in human lymphatic endothelial cells from patients with breast cancer. Lymphat Res Biol 5: 115-126, 2007.

7. Yang J, Wu HF, Qian LX, et al: Increased expression of vascular endothelial growth factor (VEGF), VEGF-C and VEGF receptor-3 in prostate cancer tissue are associated with tumor progression. Asian J Androl 8: 169-175, 2006.

8. Filho AL, Baltazar F, Bedrossian C, Michael C and Schmitt FC: Immunohistochemical expression and distribution of VEGFR-3 in malignant mesothelioma. Diagn Cytopathol 35: 786-791, 2007.

9. Saintigny P, Kambouchner M, Ly M, et al: Vascular endothelial growth factor-C and its receptor VEGFR-3 in non-small cell lung cancer: concurrent expression in cancer cells from primary tumour and metastatic lymph node. Lung Cancer 58: 205-213, 2007.

10. Van Trappen PO, Steele D, Lowe DG, et al: Expression of vascular endothelial growth factor (VEGF)-C and VEGF-D, and their receptor VEGFR-3, during different stages of cervical carcinogenesis. J Pathol 201: 544-554, 2003.

11. Su JL, Yang PC, Shih JY, et al: The VEGF-C/Flt-4 axis promotes invasion and metastasis of cancer cells. Cancer Cell 9: 209-223, 2006.

12. Timoshenko AV, Rastogi S and Lala PK: Migration-promoting role of VEGF-C and VEGF-C binding receptors in human breast cancer cells. Br J Cancer 97: 1090-1098, 2007.

13. Masood R, Kundra A, Zhu S, Xia G, Scalia P, Smith DL and Gill PS: Malignant mesothelioma growth inhibition by agents that target the VEGF and VEGF-C autocrine loops. Int J Cancer 104: 603-610, 2003.

14. Dias S, Choy M, Alitalo K and Rafii S: Vascular endothelial growth factor (VEGF)-C signaling through FLT-4 (VEGFR-3) mediates leukemic cell proliferation, survival and resistance to chemotherapy. Blood 99: 2179-2184, 2002.

15. Wong SY and Hynes RO: Tumor-lymphatic interactions in an activated stromal microenvironment. J Cell Biochem 101: 840-850, 2007. 